\title{
The World Health Organisation Disability Assessment Scale (WHODAS II): Links between self-rated health and objectively defined and clinical parameters in the population of spinal cord injury
}

\author{
V. Steinerte and A. Vetra \\ Rīga Stradinš̌ University, Riga, Latvia
}

\begin{abstract}
There are many clinical and objectively defined parameters that are used to evaluate a person's disability. Since the World Health Organisation has presented the WHODAS II as a means of objectively measuring subjectively defined functions, greater attention has been focused on self-rated health. Only a few studies, however, have been conducted about differences between self-rated health and objectively defined parameters. The survey for this study was conducted on the basis of WHODAS II and the population in Latvia with spinal cord injury. Respondents were between 18 and 65, and 98 questionnaires were analysed. The results show that people with spinal cord injury on average rate their functioning as limited (33-40 points of 100). Most respondents have been declared to be disabled, which is defined as very serious or severe functional disorders. More than $40 \%$ have paid jobs, while one-third do not work for reasons of health. The research shows that there is a close coherence $(\mathrm{p}<0.05)$ between individual, objectively and clinically defined indicators on the one hand and the aspects of the questionnaire in which physical functioning was an important factor on the other hand. In order to understand the real functional abilities of patients and the individual factors that influence those abilities, it is necessary to define functional self-rated health in addition to objectively defined indicators.
\end{abstract}

Key words: ICF, self-rated health, spinal cord injury

\section{Introduction}

The aim of rehabilitation is to help a person to regain his or her health and to minimise functional disorders. Physical, as well as mental and social functions are part of functioning, and almost each person has faced a functional disorder, whether it is short-term or still existing. This means a certain level of disability. According to the World Report on Disability, a disability is seen as a normal part of every human life. In order to eliminate functional restrictions as effectively as possible, it is important to understand and evaluate them, particularly in relation to people with disabilities who face restrictions on a daily basis [1]. One of the most common disorders that can cause long-term or permanent disability is the spinal cord injury. Such people have different physical and social barriers that influence their daily activities.

The World Health Organisation has developed the International Classification of Functioning, Disability and Health (ICF) to evaluate the individual's functional abilities. It mentions different functions, but does not provide a complete illustration of the patient's real life. It is also true that the ICF is too complicated and impractical for any medical specialist in terms of using it in clinical practice without special training [2]. As is the case with 
research in the world, the importance of self-rated health for people with chronic diseases and disorders has been stressed even more, and it has also been emphasised in the World Report on Disability. After years of hard work, the WHO has developed the self-rated health questionnaire, WHODAS II, and it is based on the ICF. Its validity and sensitivity have been demonstrated in international and intercultural studies [2], and it has been recommended for use in relation to the Latvian questionnaire, as well [3].

Researchers often find that the self-esteem that relates to functioning is often compared to other self-rated health questionnaires, but there has not been enough research on how the self-rated health of functioning differs from objectively defined indicators. In Latvia, many clinical indicators related to disability groups that reflect their functional abilities have been objectively defined for people with spinal cord injury, but this begs the question of whether people themselves evaluate their functional abilities in a similar or different way than the evaluation of medical specialists on the basis of the unified ICF mode.

The purpose of this research is to explore links between people with spinal cord injury in terms of self-rated health on the one hand and objectively defined clinical parameters on the other hand.

\section{Materials and methods}

\section{Participants}

The research cohort involves people with spinal cord injury diagnoses who, between January 2011 and August 2014, were treated at the Spinal Section of the Vaivari National Rehabilitation Centre in Latvia (NRC). Respondents were between 18 and 65 years old. Other illnesses apart from pelvic organ dysfunctions were not noted.

The exclusion criteria included diagnosed multiple sclerosis, the patient's level of activity when discharged from the rehabilitation centre and identified as "immobile" or "needing full assistance," as well as no contact number being provided.

The Ethics Commission authorised the study.

\section{The assessment tool}

The assessment tool that is used in the study is the World Health Organisation Disability Assessment Schedule (WHODAS II). The questionnaire included 36 questions in Latvian and in Russian. The WHODAS II assessment tool evaluates the functional level of life in six domains - understanding and communicating, getting around, self-care, getting along with others, life activities, and participation.

Respondents were asked to evaluate the level of difficulty related to specific activities during the previous 30 days - "None," "Mild," "Moderate," "Severe," and "Extreme or cannot do." This reflected the specific functional activities of the respondents. It took 5-10 minutes to complete the questionnaire.

The results were interpreted in accordance with the WHODAS II questionnaire manual, which includes a specific formula to calculate each domain separately and then all domains taken together. Points from 0 to 100 defined the self-rated functional health of respondents, with 0 meaning no functional restrictions, and 100 meaning total functional restrictions [2].

The study type was quantitative, cross-sectional and descriptive.

\section{Data analyses}

Microsoft Excel and SPSS 17.0 were used to process the data, with descriptive statistics and correlations. 
The Kolmogorov-Smirnov test was used to assess data distribution between the domains of the WHODAS II questionnaire and the overall indicators. The Mann-Whitney U test was used to determine whether the results of the WHODAS II domains differentiated between people with severe spinal cord injury (tetraplegia) and those with a lower level of spinal cord injury (paraplegia).

The Spearman rank correlation co-efficient was applied to search for the correlation and relationship between the WHODAS II questionnaire results and the objective defined level of disability by the respondents themselves (the disability group, the level of injury).

Cronbach's alpha co-efficient was used to check the inner consistency of the completed questionnaires, with $>0.9$ interpreted as great consistency, 0.7-0.9 as good, 0.6-0.7 as acceptable, and $<0.6$ as poor [4].

\section{Procedures in the study}

The authors posted an informational letter for potential participants, the questionnaire WHODAS II and a set of questions about the demographic situation of participants on their Google E-mail disc, also establishing a hyperlink.

A list of patients who had received rehabilitation services from the Spinal Section of the Vaivari NRC between 2011 and August 2014 was obtained. Patients who satisfied the inclusion criteria were selected. The histories of potential participants were analysed at the archive of the centre, and those who did not satisfy the criteria were excluded.

The authors rang people who satisfied the criteria, briefly explained the reason for the call, and asked whether the potential participants would like to receive an informational letter about the study and the questionnaire. Those who said yes gave their E-mail address or postal address if they did not have access to the Internet. An ID number was assigned to each patient to ensure confidentiality, and only the authors could decode that number.

\section{Results}

353 potential participants from the Vaivari NRC were selected, and 243 histories that corresponded to the criteria were found in the archive. 139 potential participants were called, five said that they did not want to receive the letter and questionnaire, three said that they were completely healthy, and in two cases it was found that the potential participant had passed away. 129 people agreed to receive the letter and the questionnaire. 111 E-mails were sent, and 18 questionnaires were sent by mail. 101 respondents responded - 84 on the Internet and 17 by mail. After the authors reviewed the completed questionnaires, they disqualified three participants who did not satisfy the requirements. Of the 98 respondents who were analysed, 27 (27.6) were women, and 71 (74.24) were men. The average age of respondents was 43.9 $( \pm 23.5)$.

According to the disease histories from the Vaivari NRC, 33 (34) patients could walk, while 65 (66) had to remain seated. Clinical and objectively defined parameters about the participants are presented in Tables 1 and 2.

40 (40.8) respondents said that they have paid jobs, while 32 (32.7) were unemployment for health reasons, and 8 (8.2) did not work for other reasons (Fig. 1).

\section{Results of the WHODAS II questionnaire}

The Cronbach $\alpha$ coefficient demonstrated good internal coherence for the completed questionnaires (0.7-0.9). In summing up the domains of the WHODAS II questionnaires, the highest score related to the domains "Life activities at home" (58.2 points), "Participation" 
Table 1. The clinical parameters of participants $(n=98)$.

\begin{tabular}{|l|l|}
\hline ASIA impairment scale, $\boldsymbol{n}(\boldsymbol{\%})$ & $18(18)$ \\
A & $33(34)$ \\
B & $30(31)$ \\
D & $13(13)$ \\
Lesion level, $\boldsymbol{n}(\boldsymbol{\%})$ & \\
Paraplegia & $55(56.1)$ \\
Tetraplegia & $43(43.9)$ \\
Cause, $\boldsymbol{n}(\boldsymbol{\%})$ & \\
Trauma & $74(75.5)$ \\
Spinal stenosis & $19(19.4)$ \\
Myelopathy & $4(4.1)$ \\
Tumour & $1(1)$ \\
\hline
\end{tabular}

Table 2. Objectively defined parameters of participants $(n=98)$.

\begin{tabular}{|l|l|}
\hline Segments, $\boldsymbol{n}$ (\%) & $7(7.1)$ \\
C1-4 & $42(42.9)$ \\
C5-Th2 & $23(23.5)$ \\
Th3-11 & $25(25.5)$ \\
Th12-S2 & $1(1.0)$ \\
S3-5 & \\
Disability level, $\boldsymbol{n}(\%)$ & $74(75.5)$ \\
High & $18(18.4)$ \\
Severe & $5(5.1)$ \\
Low & $1(1.0)$ \\
Not fixed & \\
\hline
\end{tabular}

\begin{tabular}{r|c} 
Volunteer & $2(2.0)$ \\
Student & $2(2.0)$ \\
Householder & $3(3.1)$ \\
Self-employed person & $3(3.1)$ \\
Pensioner & $8(8.2)$ \\
\hline (due to other reasons) & $8(8.2)$
\end{tabular}

Unemployed (due to other reasons) $\quad 8(8.2)$

Unemployed (due to health reasons)

Salaried work

Figure 1. Occupation of respondents, $n(\%)$.

(54.2 points), and "Getting around" (51 points). The lowest score related to "Understanding and communication" (18.4 points). On average, people with spinal cord injury in Latvia rate their functioning at a level of 40.9 points, while respondents who have jobs or are students rate it at a higher level -33.5 points.

The Kolmogorov-Smirnov test shows that in all domains apart from "Communication with people," the data correspond to a non-parametric distribution. The Cronbach $\alpha$ coefficient in all domains apart from "Communications with people" is within 0.7 and 0.9 , which means good internal coherence for the completed questionnaires.

The Mann-Whitney $U$ test shows the greatest difference between paraplegia and tetraplegia patients in several domains. As could be expected, the biggest difference related 
Table 3. Results of WHODAS II.

\begin{tabular}{|l|l|l|l|l|l|}
\hline Domain & $\mathbf{N}$ & Mean (SD) & Median & K-S $^{1}$ & Cronbach Alpha \\
\hline Understanding and communicating & 98 & $18.4(17.7)$ & 15 & 0.18 & 0.79 \\
\hline Getting around & 98 & $51.7(25.9)$ & 50 & 0.16 & 0.75 \\
\hline Self-care & 98 & $37.9(31.0)$ & 30 & 0.2 & 0.86 \\
\hline Getting along with others & 97 & $25.4(18.9)$ & 16.7 & $0.26^{*}$ & 0.63 \\
\hline Life activities at home & 98 & $58.2(30.7)$ & 50 & 0.23 & 0.88 \\
\hline Life activities at work/school & 43 & $27.4(23.3)$ & 21.4 & 0.15 & 0.9 \\
\hline Participation & 97 & $54.2(19.9)$ & 50 & 0.13 & 0.8 \\
\hline Total & 96 & $40.9(16.8)$ & 39.7 & - & 0.82 \\
\hline Total (work/school) & 43 & $33.5(15.8)$ & 29.2 & - & 0.86 \\
\hline
\end{tabular}

to domains that are related to physical functioning - "Self-care" (3.52), "Life activities" (2.141-2.243), and "Getting around" (1.560). There are no significant differences in domains related to communication, understanding and participation.

\section{Spearman correlation results}

Close coherence $(\mathrm{P}<0.05, \mathrm{p}<0.01)$ was mostly found between domains in the WHODAS II questionnaire. Most of the coherences related to domains in which physical functioning abilities were reflected - "Life activities," "Getting around," and "Self-care." The "Participation" domain correlated significantly $(\mathrm{p}<0.05)$ with all domains in the questionnaire.

Important correlations were found between the respondent's occupation and objectively and clinically defined indicators $(\mathrm{p}<0.05, \mathrm{p}<0.01)$.

Evaluating correlations between those results of the WHODAS II questionnaire which reflect functional self-evaluation and the objectively and clinically defined level of functioning of respondents, many close and important coherences were found $(\mathrm{p}<0.05$, $\mathrm{p}<0.01)$. The most important correlations were in the "Self-care" domain, which closely correlated in a negative way with the level of injury, the ASIA evaluation, the segment of injury, and the disability group. At the same time, a close and positive correlation exists between self-care and occupation (Table 2).

"Life activities at home" correlated significantly with the level of neurological injury (tetraplegia and paraplegia), as well as with occupation. "Life activities" also showed important coherence with the ASIA functional level and type of movement among those who work or study. Interestingly, there is also close coherence between the domain "Understanding and communication" and the ASIA evaluation.

Occupation correlated positively to the majority of domains that are connected to physical functioning and, of course, getting along with others.

Whereas the common results of the domains only showed poor or unimportant correlations to objectively defined and clinic indicators, there was one exception - of importance was coherence between occupation and the sum of the results of respondents who work or study.

\section{Discussion}

The data about the employment of respondents show that most of them have a paid job, work as volunteers, or are students. Only one-third reported not working for reasons of health, although most respondents have been given the disability group which, according to Latvian regulations, means severe functional limitations and disabilities. A positive factor is that a comparison of data from the questionnaires and information from the archives of the Vaivari 
Table 4. Spearman correlation results.

\begin{tabular}{|l|c|c|l|l|l|l|}
\hline $\begin{array}{l}\text { WHODAS II } \\
\text { domains }\end{array}$ & ASIA & $\begin{array}{l}\text { Lesion } \\
\text { level }\end{array}$ & Segments & $\begin{array}{l}\text { Disability } \\
\text { level }\end{array}$ & Mobility & Occupational \\
\hline $\begin{array}{l}\text { Understanding } \\
\text { and } \\
\text { communicating }\end{array}$ & $\mathbf{0 . 2 0 *}$ & -0.04 & -0.06 & -0.06 & 0.13 & -0.15 \\
\hline Getting around & -0.09 & -0.16 & -0.17 & -0.18 & 0.05 & $\mathbf{0 . 2 3}^{*}$ \\
\hline Self-care & $\mathbf{- 0 . 2 0}$ & $\mathbf{0 . 3 6}$ & $-0.24^{*}$ & $-0.21^{*}$ & -0.05 & $\mathbf{0 . 3 5}^{* *}$ \\
\hline $\begin{array}{l}\text { Getting along } \\
\text { with others }\end{array}$ & 0.09 & 0.04 & 0.01 & -0.06 & 0.02 & $\mathbf{0 . 2 3}$ \\
\hline $\begin{array}{l}\text { Life activities at } \\
\text { home }\end{array}$ & -0.07 & $-0.22^{*}$ & -0.14 & -0.10 & 0.13 & $\mathbf{0 . 2 9 *}$ \\
\hline $\begin{array}{l}\text { Life activities at } \\
\text { work/school }\end{array}$ & $\mathbf{0 . 4 4} * *$ & -0.18 & -0.14 & 0.26 & $\mathbf{0 . 3 9} * *$ & 0.07 \\
\hline Participation & 0.08 & -0.06 & -0.07 & -0.01 & 0.17 & 0.14 \\
\hline Total & 0.06 & -0.20 & -0.08 & -0.01 & 0.20 & 0.06 \\
\hline Total (work/school) & 0.00 & -0.19 & -0.18 & -0.15 & 0.11 & $\mathbf{0 . 3 0}$ \\
\hline
\end{tabular}

${ }^{* *} \mathrm{p}<0.05, * \mathrm{p}<0.001$.

NRC showed that 17 people found a paid job after being unemployed. Employment may also be based on the fact that most respondents had a higher or secondary vocational education.

When it comes to the results of WHODAS II domains, Latvians with spinal cord injuries have the greatest difficulty in terms of "Life activities" - more so at home than at work or school - as well as "Participation" and "Mobility." These results coincide with studies conducted by Van der Zee, et al. and Wolf, et al. [5.6]. An Estonian study conducted by Kivisild, et al., made use of a quality of life questionnaire and found that physical functioning and physical roles are the most limited life quality indicators for people with spinal cord injuries [7].

Respondents had reported the fewest problems with "Understanding and communication," which fully coincides with other WHODAS II questionnaire studies involving people with spinal cord injury $[5,6,8]$. The results from Latvian respondents in all domains and the evaluation of the overall level of functioning were several points higher than has been the case in studies conducted in other countries, which suggests that people with spinal cord injuries in Latvia evaluate their level of functioning lower than people in other countries do. Still, the total sum of functional evaluation points is not high enough for people with spinal cord injuries to say that their functioning is based on a very severe or severe disability, and the functional evaluation complies more with the World Report on Disability than with the definition in Latvia of what disability means.

The overall functioning evaluation correlates significantly with all life domains, particularly those in which there is an important role for physical functioning. This coincides with a study conducted by Machacova, et al., about the health and physical functioning of people with spinal cord injury [9]. Erosa, et al., by contrast found that people with spinal cord injury for whom severe functional disorders have been defined do not always evaluate their overall health status as being bad. Better participation in society is experienced by those respondents who have a better evaluation of their physical functioning (getting around, self-care, and life activities), and that corresponds to studies about the evaluation of quality of life and physical functioning [9-11].

The Cronbach alpha test results indicate that respondents filled out the questionnaire in a well-co-ordinated way and that they had a good understanding of nearly all of the questions in the various domains. A study conducted by De Wolf, et al., analysed each question in the domains, producing a table to show those answers that were unexpectedly positive or 
negative, given the respondents' objectively defined level of functioning [6]. The Cronbach alpha test showed acceptable coherence $(0.63)$ in the "Getting along with others" domain in this study.

The Mann-Whitney U test showed essential differences between the answers of people with tetraplegia and paraplegia in relation to "Self-care," "Life activities" and "Getting around," while there were no major differences in domains related to communication, understanding and participation. These results fully coincide with those from the De Wolf, et al., study [6]. It is interesting that respondents with tetraplegia self-evaluated their functioning at a much higher level (greater functional limitations) than respondents with paraplegia did, even though the disability group according to Latvian regulations was often the same.

In analysing coherences between occupations and objectively and clinically defined indicators, it is logical that people with higher disability groups have a higher ASIA evaluation level and seldom are employed at home or at a job or go to school.

Evaluating correlations between functional self-evaluation and objectively and clinically defined functional levels, important correlations $(\mathrm{p}<0.05$ and $\mathrm{p}<0.001)$ were mostly found between objectively defined indicators and those domains in which physical functioning is of importance. Significant correlations between the totalled points of functional self-evaluation and objective indicators were not identified.

Functional self-evaluation differs from expected functional limitations and physical abilities, as was noted in Machacova, et al. [9]. Functional abilities must be seen in the context of functional self-evaluation, which can be affected and influenced by a variety of individual barriers and opportunities that are faced by respondents on an everyday basis.

\section{Conclusions}

1. $90 \%$ of respondents have been assigned a disability group according to Latvian regulations that is defined as very serious or severe functional disorders. More than $40 \%$ of respondents have paid jobs, and only one-third said that they do not work for reasons of health.

2. People with spinal cord injury in Latvia, on average, rate their functioning (WHODAS II) as being limited (33-40 points of 100).

3. When it comes to the functional self-rated health (WHODAS II) of respondents with tetraplegia and paraplegia, the biggest difference relates to physical functions, while there are no differences in terms of society, understanding and communicative abilities.

4. The self-related health (WHODAS II) of respondents in terms of functions is most specifically linked to objectively and clinically defined indicators.

5. Factors that influence the functional self-evaluation of health among people with spinal cord injury are broader than is reflected by objectively and clinically defined indicators.

\section{References}

[1] World Health Organisation. World Report on Disability. Geneva, 2011. See http: //www . who. int (last viewed 7 October 2014).

[2] Üstün, T. B., Kostanjesek, N., Chatterji, S. and J. Rehm. Measuring Health and Disability: A Manual for the WHO Disability Assessment Schedule (WHODAS 2.0). World Health Organisation, 2010.

[3] A Handbook on Evaluating Functioning and Work Abilities, Latvian State Commission of Health and Work Ability Experts. See http://www. vdeavk.gov. Iv (last viewed on 20 October 2014), in Latvian. 
[4] Eisinga, R., Grotenhuis, M and B. Pelzer (2013). "The Reliability of a Two-Items Scale: Pearson, Cronbach or Spearman-Brown?", International Journal of Public Health, 58(4): 637-642.

[5] Van der Zee, C. H., Post, M. W., Brinkhof, M. W. and R. C. Wagenaar. "Comparison of the Utrecht Scale for Evaluation of Rehabilitation-Participation with the ICF Measure of Participation and Activities Screener and the WHO Disability Assessment Schedule II in Persons With Spinal Cord Injury, Archives of Physical and Medical Rehabilitation, January 2014; 95(1): 87-93.

[6] Wolf, A. C., Tate, R. L., Lannin, N. A., Middleton, J., Lane-Brown, A., and I. D. Cameron. "The World Health Organisation Disability Assessment Scale, WHODAS II: Reliability and Validity in the Measurement of Activity and Participation in a Spinal Cord Injury Population," Journal of Rehabilitative Medicine, September 2012; 44(9): 747-755.

[7] Kivisild, A., Sabre, L., Tomberg, T., Ruus, T., Kõrv, J., Asser, T. and U. Linnamägi. "Health-Related Quality of Life in Patients with Traumatic Spinal Cord Injury in Estonia," Spinal Cord, July 2014; 52(7): 570-575.

[8] Post, M. W., de Witte, L. P., Reichrath, E., Verdonschot, M. M., Wijlhuizen, G. J. and R. J. Perenboom. "Development and Validation of IMPACT-S, an ICF-Based Questionnaire to Measure Activities and Participation," Journal of Rehabilitative Medicine, August 2008; 40(8): 620-627.

[9] Machacova, K., Lysack, C. and S. Neufeld. "Self-Rated Health Among Persons with Spinal Cord Injury: What is the Role of Physical Ability?", Journal of Spinal Cord Medicine, 2011; 34(3): 265-272.

[10] Simpson, G. and K. Junes. "How Important is Resilience Among Family Members Supporting Relatives with Traumatic Brain Injury or Spinal Cord Injury?", Clinical Rehabilitation, April 2013; 27(4): 367-377.

[11] Erosa, N. A., Berry, J. W., Elliott, T. R., Underhill, A. T. and P. R. Fine. "Predicting Quality of Life 5 Years After Medical Discharge for Traumatic Spinal Cord Injury," British Journal of Health Psychology, November 2014; 19(4): 688-700. 